\title{
Health Engagement Living Lab Project: A Pilot Mixed-Methods Study To Advance Self-Care Agency Among Migrant Workers In South Korea
}

Youlim Kim

Mo-Im Kim Nursing Research Institute, College of Nursing and Brain Korea 21 FOUR Project, Yonsei University

Hyeonkyeong Lee ( $\sim$ hlee39@yuhs.ac )

Mo-Im Kim Nursing Research Institute, College of Nursing and Brain Korea 21 FOUR Project, Yonsei University

Sookyung Kim

Yonsei University College of Nursing

Junghee Kim

Yonsei University Wonju College of Medicine, Department of Nursing

\section{Research Article}

Keywords: migrant, health promotion, self-care agency, mixed methods study, living lab

Posted Date: April 28th, 2021

DOI: https://doi.org/10.21203/rs.3.rs-438549/v1

License: (a) (1) This work is licensed under a Creative Commons Attribution 4.0 International License. Read Full License 


\section{Abstract}

Background: Migrant workers struggle to access health services and adapt to a new culture. Self-care agency can be important for health self-management. It is necessary to investigate in detail how migrant workers engage in health promotion behaviors. This study examines the effects of a health engagement program to improve self-care agency using a living lab approach among migrant workers living in South Korea.

Methods: Participants included 42 migrant workers from 9 countries, recruited via flyers, posters, and internet bulletins posted by a migrant community organization. This pilot study used a mixed-methods design including a nonequivalent pre-post-test control group and three focus group interviews. During the 12-week period, only the intervention group participants received four workshops addressing healthy physical activity, healthy dietary habits, effective cultural adaptation, stress management, and two outdoor cultural activities. They also participated in focus group interviews after the second, third, and fourth group activities and discussed the benefits of health behaviors and specific ways to implement them in real life. The control group received no intervention.

Results: After the 12-week intervention period, participants in the intervention group showed an increase in the levels of self-care agency $(U=82.5, p<.001)$, health literacy $(U=91.5, p<.001)$, and acculturation $(U=119.0, p=.010)$ compared to the control group. As results of qualitative content analysis, various themes were derived for three areas: healthy diet, acculturation, and stress management based on lessons learned from living lab activities, barriers to health behavior practices and methods to overcome barriers.

Conclusions: This study demonstrated that interventions using a living lab were effective in increasing migrants' participation in health promotion activities by strengthening their self-care agency. The qualitative approach was useful in obtaining more comprehensive results on strategies aimed at enhancing health behavior engagement.

\section{Background}

International migration is a worldwide phenomenon, and the various health issues of migrants have become a significant public health concern [1]. A study of health care behaviors and primary care among migrants found that approximately $30 \%$ of respondents reported problems with self-care activities, such as taking prescribed medications, exercising regularly, and monitoring health [2]. Many migrant workers did not receive safety and health education, even though most experience musculoskeletal health problems due to long hours of excessive physical exertion [3]. Additionally, more than half (58.1\%) of workers reported they did not exercise on a regular basis, placing them at an increased risk of health problems [3], and nearly $64 \%$ of survey participants were classified as overweight or obese in relation to high fast food intake rates [4]. Along with physical health issues, migrant workers with low sociocultural adaptation competency were more likely to have poorer mental health [5]. Therefore, active health care and community adaptation are crucial for migrant workers.

Self-care agency is defined as the operational capacity that directly influences self-management for health promotion [6]. Because migrant physical and psychological outcomes can be negatively affected by cultural differences and cultural adaptation difficulties, self-care agency can be important for migrants if they wish to develop effective management and health-promotion skills [2].

Acculturation is a multidimensional cultural and psychological change process that results from a contact between two or more different cultures and groups [7]. A high level of acculturation is associated with positive aspects such as improved language ability, higher socioeconomic status, easy access to health care and preventive health services, lower depressive symptoms, and better quality of life and health outcomes for migrants in general $[8,9]$.

Social support is another key factor that reduces acculturative stress, depression, and anxiety levels, and positively affects psychological well-being among migrants [10]. Furthermore, social support that arises from active participation in host community activities is known to promote a sense of community among migrants [11]. The WHO called on improving health literacy, which has been identified as one of the social determinants of health [12], including enhancing the understanding, knowledge, evaluation, and application of health information for health care-related decision-making, disease prevention, and health 
promotion. Understanding and appropriately applying health information may result in better self-care [13]. As migrant workers in Korea have lower health literacy than native Koreans, they have difficulty addressing their own health problems and making independent decisions to improve their health status, thereby greatly increasing risk of physical and mental health issues [14].

Some intervention programs, developed to promote health among Korea's migrant workers, are focused on psychosocial outcomes [15]. Mucci et al. [16] reviewed current intervention programs for migrant workers and found that these were primarily focused on safety education in the workplace, health promotion, such as walking and stretching exercises, or disease prevention, such as prevention of malaria and sexually transmitted infections [17]. However, only a limited number of studies focus on self-care efficacy. It is important to encourage in health-promoting behavior. The living lab approach is an innovative way of identifying engagement strategies by brainstorming with participants [17]. It is an open innovative experimental environment where users act as a leading player rather than just a participant in a real-life setting, and it allows users to participate in the entire process from experimental design to evaluation to provide a new perspective [18]. It is also necessary to investigate in detail how migrant workers engage in health promotion behavior. Mixed-methods research practices that combine quantitative and qualitative evaluation approaches thus provide a better understanding of research outcomes than either method would do alone [19]. Thus, mixed methods are more appropriate in exploring the effects of the living lab interventions that co-create interactions among multiple stakeholders.

The purpose of this study was to examine the effects of a health engagement program, which applied the six principles (multimethod approach, users' engagement, multi-stakeholder participants, real-life settings, co-creation, and innovation activity) of the living lab [20] to improve self-care agency among 42 migrant workers.

\section{Materials And Methods}

\section{Study design}

This study used a concurrently embedded mixed-method design combining quantitative and qualitative research (Fig. 1). This was conducted as a pilot study; quantitative research was carried out using a nonequivalent pre-post-test control group, and qualitative research was done through focus group interviews.

\section{Study sample}

Participants were recruited through invitations on flyers, posters, and internet bulletins of social welfare institutes for migrant workers. The inclusion criteria were migrant workers, who were legally employed adults under 40 years of age, working full-time, with intermediate-level Korean or Level 3 or higher qualification in the Test of Proficiency in Korean (TOPIK), a smart cellular phone, and the ability to use mobile applications. TOPIK Level 3 is equivalent to intermediate-level Korean language proficiency, which makes it possible to perform basic language functions necessary for using various public facilities and maintaining social relations without difficulty. Illegal migrants and those who had participated in other health promotion programs in the preceding three months were excluded. We contacted 66 migrant workers who showed interest in this study but 19 did not meet the inclusion criteria. A total of 47 migrant workers from 9 countries (i.e., Cambodia, Mongolia, Vietnam, Myanmar, etc.), 25 in the intervention group and 22 in the control group agreed to participate in 12 intervention weeks. During the intervention, five dropped out and the final analysis included data from 42 workers, 21 in each of the two groups.

\section{Intervention}

During the 12-week intervention period, a total of six sessions, consisting of four group activities (healthy physical activity, healthy dietary habits, acculturation, and stress management) in the form of workshops involving discussion and practice, and two cultural activities (university tour and dental check-up, marathon participation in a community sports event) in the form of outdoor activities using community resources were organized. All sessions were based on living lab principles, which encourage participants to solve their own health problems themselves. The first session on "Healthy physical activities" consisted of stretching and muscle exercises in accordance with the 2014 Musculoskeletal Disease Prevention Manual of the Korea Occupational Safety and Health Agency. Participants learned stretching and muscle exercise movements followed by a discussion about their benefits and specific ways to implement them in real life. Second, the session on "Healthy dietary habits" involved the 
dissemination of information on intake of a low-sodium diet to help cultivate healthy dietary habits. Participants assessed their taste for salt intake with sample foods and were invited to discuss their usual dietary behaviors and healthy dietary practices. In addition, participants were asked to consult the Ministry of Food and Drug Safety website to find restaurants that served low sodium meals in their neighborhood. The third group activity session stimulated participants' interest in Korean cultural events and enhance access to community resources by introducing 80 community programs to enable early adaptation and assimilation of migrant workers into Korean society. Lastly, participants were introduced to stress management techniques based on a better understanding of their personalities and that of others using DISC, a test tool for understanding propensity and useful for classifying behavior into four personality types (i.e., Dominant, Influencer, Steady, and Conscientious) so as to improve communication methods with peers of different personality types [21]. At the end, participants were able to identify and understand their own personality type and that of the others; how migrant workers can communicate better in order to establish good interpersonal relationships was also discussed.

The contents and location of the cultural activities were decided based on participants' preferences. First, migrant workers were offered a tour of a private university campus, from where the researchers hailed, and informed about its history and culture. By participating in a free dental care program at the university hospital, they were instructed in the importance of regular dental care. Second, a marathon along the Han River was organized; by participating in a $5 \mathrm{~km}$ marathon together, participants and researchers were able to appreciate the benefits of community resources used to organize physical activities and cultural events.

\section{Study Measures}

\section{Health promotion lifestyle}

The health promotion lifestyle profile (HPLP) was developed by Walker et al. [22] and originally comprised 26 items but was later shortened by Jeon et al. [23]. The HPLP uses a four-point Likert scale ( $1=n e v e r$, and $4=$ routinely), with higher total scores representing better lifestyle conditions for improving health. Cronbach's a was .70 in this study.

\section{Health literacy}

The health literacy scale was developed by An and Yang [24] to measure the ability of migrant married women to read, write, understand, and use health information. The questionnaire comprises 10 items rated on a five-point Likert scale $(0=s t r o n g l y$ disagree, and $4=$ strongly agree). The mean level of health literacy in migrant workers and that of migrant married women (original study cohort) with intermediate-level Korean were found to be similar. Cronbach's a was .71 in this study.

\section{Self-care agency}

Self-care agency was measured using a questionnaire developed by Evers et al. [25] and translated by Kim. [26] It comprised a total of 15 items with responses ranging on a five-point Likert scale ( $1=$ strongly disagree, and $5=$ strongly agree). Scores for selfcare agency were directly proportional to the total score. Cronbach's a was .70 in this study.

\section{Sense of Community}

Sense of community was measured using the Korean version of the Brief Sense of Community Scale (BSCS), which was based on Peterson and colleague's [27] scale and modified by Oh et al. [28]. The scale includes eight items, rated on a five- point Likert scale ( 1 = strongly disagree, and 5 = strongly agree). Some of the BSCS items were changed for the purposes of this study: for e.g., "peer/friend" to "neighborhood" and "peer group" to "neighbor." The sense of community score was directly proportional to the total score. Cronbach's a was .87 in this study.

\section{Social support}

Social support was measured using a scale developed by Zimet et al. [29] and reconstructed by Shin and Lee [30]. This scale consists of 12 items: there were 4 questions each on support from the family, support from meaningful others, and support from friends. Each response was measured on a five-point Likert scale ( $1=$ strongly disagree, and $5=$ strongly agree $)$. Scores for social support were directly proportional to the total score. Cronbach's a was .88 in this study.

\section{Acculturation}


Acculturation was measured using a questionnaire based on Barry's East Asian Acculturation Measurement and modified by Jang [31]. This scale has 10 items measured on a five-point Likert scale $(1=$ strongly disagree, and $5=$ strongly agree). The acculturation level was directly proportional to the total score. Cronbach's a was .72 in this study.

\section{Focus group interviews}

Focus group interviews were conducted to freely discuss individual experiences, perceptions, and knowledge related to the intervention through active interaction with participants. It included an opening question, introductory question, transitional question, key questions, and a final question. Key questions were: "What are the useful conjectures found in today's health community activity?" "What are the barriers to engaging in health behavior in your daily life?" and "How can we overcome barriers and engage in health behaviors?"

\section{Data collection}

This study was conducted in accordance with the guidelines set out in the Declaration of Helsinki, and all procedures involving study participants were approved by the ethics committee of the University of Yonsei. This study was conducted from June to September 2019. Researchers trained in ethics explained the study purpose, method, and process to participants before data collection; written informed consent was provided by all the participants.

All participants participated in two surveys: pre-test and post-test, and quantitative data were collected using self-reported questionnaires. Participants who did not understand the questionnaire were provided explanations in person by trained research assistants. Qualitative data were collected on aspects not measured by the survey to supplement the results of the questionnaire. Interviews were conducted in three 40-minute rounds by doctoral students trained in qualitative research methodology. Since migrant workers have slower speech delivery in Korean, interview data were not recorded, but transcribed on the spot.

\section{Data analysis}

Quantitative data were analyzed using SPSS Statistics 25.0 (IBM Corp, Armonk, NY, USA). All continuous variables were described as mean \pm standard deviation, and categorical variables as frequency. Group differences were analyzed using the independent $t$ test, Mann-Whitney's U-test and the chi-squared test or Fisher's exact test. The pre- and post- intervention scores were compared and analyzed using Wilcoxon's signed rank test.

Qualitative data were analyzed using qualitative content analysis. The two researchers (Y.K \& J.K) read and verified interview transcripts to study migrants' health engagement experience and their willingness to participate in the health behavior program. Meaningful data from the transcripts were extracted, compared, contrasted, and classified, and the main concepts and themes derived. Additional themes were drawn up to check the validity of the analytical methods used and results were further reviewed by one nursing professor with experience in qualitative research. The themes that emerged from the analysis were agreed upon after discussion between the researchers.

\section{Results}

\section{Participants}

Table 1 gives the general characteristics of participants. The mean age of the intervention group and controls was $29.52 \pm 3.71$ years and $30.67 \pm 4.88$ years, respectively $(p=.363)$. The proportion of males was higher in both groups $(p=.513)$. Both groups had a high percentage of participants from countries in the WHO Western Pacific Region (i.e., Cambodia, Mongolia, Vietnam). The mean duration of their stay in Korea was $59.57 \pm 39.49$ months in the intervention group and $57.86 \pm 37.13$ months in the control group $(p=.950)$. The mean number of months worked at the current job was $43.14 \pm 23.95$ in the intervention group and $37.95 \pm$ 25.35 in the control group ( $p=.339)$. The mean time spent working per day was $8.95 \pm 1.99$ hours in the intervention group and $9.12 \pm 1.45$ hours in the control group $(p=.629)$. The number of graduates with more than high school education was 18 (85.7\%) in the intervention group and $21(100.0 \%)$ in the control group $(p=.072)$. In terms of type of job, skilled labor accounted for the largest proportion in both groups. Most participants in both groups were unmarried. Only 1 participant in the intervention group $(4.8 \%)$, and $2(9.6 \%)$ in the control group smoked $(p=.747)$. In terms of alcohol intake frequency, the number participants who 
consumed less than two glasses per months formed the largest proportion in both groups. There was no statistically significant difference in general characteristics between the intervention group and the control group (Table 1).

Table 1. General Characteristics of Participants $(N=42)$

\begin{tabular}{|c|c|c|c|c|c|c|c|}
\hline \multirow[t]{2}{*}{ Demographics } & \multicolumn{2}{|c|}{ Intervention $(N=21)$} & \multicolumn{2}{|c|}{ Control $(N=21)$} & \multirow[b]{2}{*}{$U$} & \multirow[b]{2}{*}{$\mathrm{t}$} & \multirow[b]{2}{*}{$p$} \\
\hline & $\mathrm{N}(\%)$ & $\operatorname{Mean}(S D)$ & N (\%) & $\operatorname{Mean}(S D)$ & & & \\
\hline Age & & 29.52(3.71) & & $30.67(4.88)$ & 184.5 & & .363 \\
\hline Sex & & & & & & 0.43 & .513 \\
\hline Male & 13(6.19) & & 15(71.4) & & & & \\
\hline Region & & & & & & 0.11 & .739 \\
\hline Western pacific & 14(66.7) & & 15(71.4) & & & & \\
\hline Southeast & 7(33.3) & & 6(28.6) & & & & \\
\hline $\begin{array}{l}\text { Duration of stay } \\
\text { (months) }\end{array}$ & & $59.57(39.49)$ & & $57.86(37.13)$ & 218.0 & & .950 \\
\hline Duration of job (months) & & 43.14(23.95) & & $37.95(25.35)$ & 182.5 & & .339 \\
\hline Working time (hr/day) & & 8.95(1.99) & & $9.12(1.45)$ & 203.0 & & .629 \\
\hline Education & & & & & & 3.23 & .072 \\
\hline$\geq$ High school & 18(85.7) & & $21(100.0)$ & & & & \\
\hline Type of job & & & & & & 1.97 & .417 \\
\hline Office & $3(14.3)$ & & $5(23.8)$ & & & & \\
\hline Skilled labor & $17(81.0)$ & & $13(61.9)$ & & & & \\
\hline Others & $1(4.8)$ & & $3(14.3)$ & & & & \\
\hline Marital status & & & & & & 0.39 & .533 \\
\hline Married & $8(38.1)$ & & $10(47.6)$ & & & & \\
\hline Smoking & $1(4.8)$ & & $2(9.6)$ & & & 2.22 & .737 \\
\hline Alcohol intake frequency /months & & & & & & 2.22 & .528 \\
\hline $1-2$ times & $10(47.6)$ & & $10(47.6)$ & & & & \\
\hline 3-4 times & $6(28.6)$ & & 7(33.3) & & & & \\
\hline$>5$ times & $5(23.8)$ & & $4(19.0)$ & & & & \\
\hline
\end{tabular}

\section{Comparison of changes between groups}

Table 2 shows the mean scores for health promotion lifestyle, self-care agency, health literacy, social support, sense of community and acculturation from baseline to Week 12 in both groups. Self-care agency scores at Week 12 compared to baseline were significantly increased in the intervention group compared to the control group $(U=82.5, p<.001)$. Compared to the control group, health literacy and acculturation scores over the 12 weeks were significantly increased in the intervention group $(U=91.5, p<.001$; $\mathrm{U}=119.0, p=.01$, respectively). However, changes in health promotion lifestyles, social support, and sense of community scores in the two groups were not significant. It should be noted that in the control group, the level of sense of community at Week 12 was significantly increased compared to baseline $(Z=2.79, p=.005)$.

Table 2. Comparison of Outcome Variables Between Groups 


\begin{tabular}{|c|c|c|c|c|c|c|c|c|c|c|}
\hline \multirow[t]{3}{*}{ Variables } & \multicolumn{4}{|c|}{ Intervention $(N=21)$} & \multicolumn{4}{|c|}{ Control $(N=21)$} & \multicolumn{2}{|c|}{ Differences } \\
\hline & \multicolumn{4}{|l|}{$\operatorname{Mean}(S D)$} & \multicolumn{6}{|l|}{$\operatorname{Mean}(S D)$} \\
\hline & Pre & Post & Z & $p$ & Pre & Post & Z & $\mathrm{p}$ & $\mathrm{U}$ & $p$ \\
\hline $\begin{array}{l}\text { Health } \\
\text { promotion } \\
\text { lifestyle }\end{array}$ & $77.19(8.54)$ & $80.71(7.89)$ & 2.247 & .025 & 77.10(8.32) & 78.33(6.81) & 1.795 & .073 & 156.0 & .103 \\
\hline $\begin{array}{l}\text { Self-care } \\
\text { agency }\end{array}$ & $51.24(5.13)$ & $55.57(5.62)$ & 3.386 & $<.001$ & $53.62(6.71)$ & $52.90(6.17)$ & 0.528 & .598 & 82.5 & $<.001$ \\
\hline $\begin{array}{l}\text { Health } \\
\text { literacy }\end{array}$ & $21.00(6.63)$ & $24.71(5.24)$ & 2.859 & .004 & $24.36(4.25)$ & $23.68(4.33)$ & 0.750 & .454 & 91.5 & $<.001$ \\
\hline $\begin{array}{l}\text { Social } \\
\text { support }\end{array}$ & $46.81(7.58)$ & $47.62(6.48)$ & 0.262 & .794 & $47.76(8.06)$ & $48.86(6.43)$ & 1.442 & .149 & 199.5 & .584 \\
\hline $\begin{array}{l}\text { Sense of } \\
\text { community }\end{array}$ & $25.33(8.64)$ & $29.71(6.50)$ & 2.710 & .007 & $22.48(6.23)$ & $24.90(5.14)$ & 2.790 & .005 & 193.0 & .484 \\
\hline Acculturation & $3.02(0.67)$ & $3.23(0.50)$ & 2.383 & .017 & $2.96(0.36)$ & $2.93(0.38)$ & 0.159 & .873 & 119.0 & .010 \\
\hline
\end{tabular}

\section{Qualitative content analysis of the health engagement intervention}

Various themes were derived based on responses to key questions used in the three focus group discussions with participants in the intervention group (Fig. 2). Topics covered by the three group activities included healthy diet, acculturation, and stress management. For each activity, themes were derived based on lessons learned from living lab activities, barriers to health behavior practices and methods to overcome these.

\section{Healthy diet}

Those who participated in activities on healthy diets said that they learned how to estimate their sodium intake using the sodium preference assessment test and had understood the negative health effects of excessive sodium intake. The following themes emerged from this activity: "Recognition of the general preference for salty foods," "Identification of the health risks due to dietary imbalances" and "Necessity to check nutritional information to ensure the health of the family." Fig. 2 shows the specific themes derived.

\section{Acculturation}

Those who participated in cultural adaptation activities found instructions on Korean culture and the use of community resources such as parks to be novel experiences. They said that this information was necessary for foreigners to adapt. They expressed their acceptance of Korean life and culture. The theme derived from this activity was "Acceptance of and assimilation with Korean culture."

\section{Stress management}

Participants said that their knowledge of personality types identified through personality tests would be useful in seeking suitable jobs. They discussed the need to understand other people's personalities in order to maintain good interpersonal relationships. The following themes emerged "Understanding of one's personality" and "Respect for other people's personalities."

\section{Healthy diet}

Participants had no previous awareness about the importance of nutritional information, and those who lived alone mostly avoided cooking by frequently eating out or ordering food. They also said that poor eating habits formed in childhood interfered with healthy eating practices in adulthood. Themes that emerged from this activity were "Lack of education regarding and awareness of nutritional information," "Korean habit of eating out or having delivered food" and "Unhealthy eating habits."

\section{Acculturation}


Most participants said that the Han River Marathon was their first experience of participating in festivals or community events in Korea. Participants showed interest in Korean cultural life and in using various community resources but said access was difficult due to lack of information. Participants used the internet to find information on Korean culture but expressed difficulty with search methods and using tools like the Internet homepage. Based on these, "Lack of accessibility to information on community resources" and "Difficulty in acculturation due to lack of searching ability" were identified as barriers to cultural adaptation of migrants.

\section{Stress management}

Participants explained that they experienced stress due to conflicts with differing personalities, especially when they tried to force others to come around to their view. The theme of "Lack of respect for other people's personalities" was identified.

\section{Healthy diet}

Participants said it was important to prepare healthy foods to ensure the health of their families and children. Participants also discussed the importance of improving Korean language skills to read about and prepare healthy food, and inform others of their preference for healthy meals. "Willingness to cook healthy meals," "Improving literacy for healthy eating" and "Changing behavior for healthy eating" were identified as themes.

\section{Acculturation}

Participants agreed they needed to enjoy Korean festivals and other cultural events with participants to increase cultural adaptation. Access to Korean cultural life could be increased by using high-powered social networking services such as Facebook. "Acculturation for greater enjoyment of Korean life" and "Using various resources on Korean cultural life" were identified as themes.

\section{Stress management}

Participants showed a willingness to overcome personal deficiencies guided by their personality test results. They also expressed the need to think positively and have greater self-confidence in order to reduce personal stress in a new and unfamiliar environment. Other participants suggested that emotional support through dialogue and humor could help face difficult situations. "Understanding of one's personality and willingness to change attitudes," "Being positive and having confidence," "Being understanding toward others," and "Providing social support to others" were identified as themes.

\section{Discussion}

\section{Principle findings}

This study examined the effects of a health engagement intervention using living lab principles among migrant workers. In the intervention group, self-care agency levels, health literacy, and acculturation at week 12 were significantly increased compared to the control group, indicating that the health engagement intervention was effective.

Living lab principles were applied as intervention strategies in this study to encourage in-depth exploration and active participation of migrant workers in health behavior engagement. Hard-to-reach populations such as migrants and disabled people who are reported to be alienated from normal communication channels for various reasons [17] generally tend to be hesitant about participating in health programs [32]. We proposed realistically possible health behaviors using a methodological guide for Hardto-reach groups $[17,32]$ and provided positive feedback. Ultimately, participation intended to help increase self-care agency in migrant workers and acculturation in Korea. In another study centered on marginalized groups in the community, the living lab method was found to increase health and media literacy and resolve health disparities through cooperation with various stakeholders [33].

Combining mixed methods with action research methods such as living lab and intervention assessment yielded credible and valid conclusions about the results of the intervention and maximized the potential of the study. Had only quantitative research methods been used, identification of the various experiences and attitudes of the health promotion activities among migrant workers would have been limited. Focus group interviews were used to discuss health issues; these discussions also provided co- 
learning opportunities and enabled multi-dimensional dynamics such as behavior motivation and results. The results of our qualitative research could be used in future studies to help plan interventions on health promoting behaviors.

Migrant workers play an important role in the labor market. Unlike domestic workers, migrant workers in Korea are often employed in small workplaces and have a high rate of anemia due to nutritional imbalances and living in suburban dormitories [34]. They are vulnerable to mental health problems, such as stress, anxiety, and loneliness, which are barriers to self-care [35]. Despite the health disparities between migrant workers and native workers, to our knowledge, no studies on interventions to increase self-care agency for health promotion have been published. In other countries, previous interventions for self-care in migrant workers mainly involved self-management of individuals with diseases such as medication adherence and self-monitoring of blood glucose levels in type 2 diabetes patients [36, 37]. Interventions for migrant workers with limited access to health information and services at work need to be designed in collaboration with workplaces and communities in accordance with the goal of "strengthening workplace cooperation for safe and healthy work" (SDG 8; United Nations, 2019) [38].

Our study results showed the intervention increased access to community information and resources, such as social and health services in the host country, and also increased cultural adaptation. A supportive social environment, with opportunities for cultural activities and recreation, as well as a comfortable physical environment, would promote acculturation and health in migrants, so that they have greater control over their own health [39]. Participating in host country cultural activities, such as sporting or social events [40] is also a positive predictor of wellbeing, as migrants may feel a greater sense of assimilation within the host society.

\section{Study Limitations}

Participants lacked the dynamism to recognize health issues and find solutions to achieve a healthy lifestyle. This might be due to language barriers and a lack of acculturation. Although utilizing a mixed methods approach was useful to identify significant and meaningful results that reflect the unique characteristics of participants, the group discussions after each intervention activity did not enable all participants to share their beliefs and values regarding health and their commitment to healthy lifestyle behaviors. Considering these limitations, future researchers should perhaps develop separate interventions by ethnic group with people who speak the same language for a sufficient time period and collaborate with interventionists with bilingual and bicultural competency.

\section{Conclusion}

Living lab methods were used in combination with mixed research methodologies in the community to create effective strategies for encouraging migrant workers to take self-health decisions and invite self-management. In order to reduce the gap in health status between Koreans and migrant workers, it is necessary to develop long-term health promotion programs with strengthened community networks in cooperation with relevant community agencies.

\section{Abbreviations}

Test of Proficiency in Korean (TOPIK)

Dominant, Influencer, Steady, Conscientious (DISC)

Health promotion lifestyle profile (HPLP)

Brief Sense of Community Scale (BSCS)

\section{Declarations}

Ethics approval and consent to participate: Ethical approval was obtained from the ethics committee of the University of Yonsei, Seoul, Korea (Y-2019-0019). All procedures of study were performed in accordance with the Declaration of Helsinki. In accordance with the ethical guidelines, written informed consent was obtained from all participants after the researchers' research explanation. Moreover, they were guided that all data would be kept confidential and would not be revealed unless for research 
purposes. Participants' identities were collected anonymously, and they were informed to withdraw from the program at any time if they wished.

Consent for publication: Not applicable

Availability of data and materials: The data that support the findings of this study are available from the corresponding author, upon reasonable request.

Competing interests: The authors declare that they have no competing interests.

Funding: This work was supported by the National Research Foundation of Korea (NRF) grant funded by the Korea government (NRF-2017R1A2B4008671, 2020R11A2069894) and Brain Korea 21 FOUR Project funded by National Research Foundation (NRF) of Korea, Yonsei University College of Nursing.

Acknowledgements: Not applicable

Authors' contributions: Y.K and H.L conceived the ideas; Y.K collected and the data; Y.K, S.K, J.K and H.L analyzed the data and advised the revision of idea; Y.K and H.L contributed to the writing of the manuscript. All authors read and approved the final manuscript.

\section{ORCID Numbers}

Youlim Kim (https://orcid.org/0000-0002-4477-2489),

Hyeonkyeong Lee (https://orcid.org/0000-0001-9558-7737),

Sookyung Kim (https://orcid.org/0000-0001-6364-6907),

Junghee Kim (https://orcid.org/0000-0002-6527-2678)

\section{References}

1. Zimmerman C, Kiss L, Hossain M. Migration and health: a framework for 21 st century policy-making. PLoS Med. 2011;8:e1001034.

2. Moreno G, Morales LS, Batts F, Noguera C, Isiordia M, Mangione CM. Migration, health care behaviors, and primary care for rural Latinos with diabetes. J Immigr Minor Health. 2016;18(2):1247-52.

3. Jung HS, Kim YK, Kim HL, Yi G, Song YE, Kim JH, et al. The health status and occupational characteristics related to gender of migrant worker in Korea. Korean J Occup Health Nurs. 2008;17(2):126-37.

4. Hall BJ, Huang L, Yi G, Latkin C. Fast Food Restaurant Density and Weight Status: A Spatial Analysis among Filipina Migrant Workers in Macao (SAR), People's Republic of China. Soc Sci Med. 2020:113192.

5. Nugraha S, Hirano Y, Sumihisha H. The change in mental health status of Indonesian health care migrant worker in Japan. Kesmas Nat Pub Health J. 2017;12(2):53-9.

6. Orem DE, Taylor SG, Renpenning KM. Nursing: Concepts of practice. St. Louis: Mosby; 2001.

7. Berry JW. Acculturation: Living successfully in two cultures. Int J Intercult Relat. 2005;29(6):697-712.

8. Lim JW, Yi J, Zebrack B. Acculturation, social support, and quality of life for Korean immigrant breast and gynecological cancer survivors. Ethn Health. 2008;13(3):243-60.

9. López L, Grant RW, Marceau L, Piccolo R, McKinlay JB, Meigs JB. Association of acculturation and health literacy with prevalent dysglycemia and diabetes control among Latinos in the Boston area Community Health (BACH) survey. J Immigr Minor Health. 2015;18(6):1266-73.

10. Ayers JW, Hofstetter CR, Usita P, Irvin VL, Kang S, Hovell MF. Sorting out the competing effects of acculturation, immigrant stress \& social support on depression: a report on Korean women in California. J Nerv Ment Dis. 2009;197(10):742-7.

Page $10 / 14$ 
11. Bostean G, Gillespie BJ. Acculturation, acculturative stressors, and family relationships among Latina/o immigrants. Cult Divers Ethnic Minor Psychol. 2018;24(1):126-38.

12. Sørensen K, Van den Broucke S, Fullam J, Doyle G, Pelikan J, Slonska Z, et al. Health literacy and public health: a systematic review and integration of definitions and models. BMC Pub Health 2012;12(1):80.

13. Kim J. Measuring the level of health literacy and influence factors: Targeting the visitors of a university hospital's outpatient clinic. J Korean Clin Nurs Res. 2011;17(1):27-34.

14. Lee JM, Lee E. Factors influencing level of health literacy of migrant workers in Korea. J Korean Acad Fundam Nurs. 2013;20(3):269-77.

15. Kim J, Park SJ, Lee J. Effects of intervention for health promotion of foreign workers: A systematic review. J Korea Converg Soc. 2018;9(9):407-20.

16. Mucci N, Traversini V, Giorgi G, Garzaro G, Fiz-Perez J, Campagna M, et al. Migrant workers and physical health: An umbrella review. Sustainability. 2019;11(1):232.

17. Pappers J, Keserü I, Macharis C. Report on the methodology for the inclusion of hard-to-reach groups. 2018. http://looperproject.eu/wp-content/uploads/2018/10/LOOPER_D3.2_Hard_to_Reach_Groups_FINAL.pdf Accessed May 6 2020.

18. Dell'Era C, Landoni P. Living lab: A methodology between user-centred design and participatory design. Creativity Innov Manag. 2014;23(2):137-54.

19. O'Cathain A, Murphy E, Nicholl J. Integration and publications as indicators of "yield" from mixed methods studies. J Mixed Methods Res. 2007;1(2):147-63.

20. Kim J, Kim YL, Jang H, Cho M, Lee M, Kim J, et al. Living labs for health: an integrative literature review. Eur J Pub Health. 2020;30(1):55-63.

21. Slowikowski MK. Using the DISC behavioral instrument to guide leadership and communication. AORN J. 2005;82(5):835-43.

22. Walker SN, Sechrist KR, Pender NJ. Health promotion model-instruments to measure health promoting lifestyle: Healthpromoting lifestyle profile [HPLP II] (Adult version), 1995;22:796-811. University of Michigan. Available from: https://deepblue.lib.umich.edu/handle/2027.42/85349. Accessed 10 March, 2019.

23. Jeon EY, Choi SR, Han SS. Influencing factors on health-promotion lifestyle of nurses. J East-West Nurs Res. 2007;13(1):40-7.

24. An J, Yang SJ. Development of a health literacy assessment scale for Asian immigrant women in South Korea. J Korean Acad Community Health Nurs. 2015;26(4):330-41.

25. Evers G, Isenberg M, Philipsen H, Brouns G, Halfens R, Smeets H. The appraisal of self-care agency's ASA-Scale: research program to test reliability and validity. In: Proceedings of the International Nursing Research Conference "New Frontiers in Nursing Research." Edmonton University of Alberta, Canada; 1986.

26. Kim BJ, Sangalang CC, Kihl T. Effects of acculturation and social network support on depression among elderly Korean immigrants. Aging Ment Health. 2012;16(6):787-94.

27. Peterson NA, Speer PW, McMillan DW. Validation of a brief sense of community scale: Confirmation of the principal theory of sense of community. J Community Psychol. 2008;36(1):61-73.

28. Oh J, Kim C, Ha MA. Study on effect of adolescents' leisure satisfaction on self-esteem and sense of community: Focused on vocational high school students. Int. J. Cult. 2012;26(2):23-40.

29. Zimet GD, Dahlem NW, Zimet SG, Farley GK. The multidimensional scale of perceived social support. Journal of Personality Assessment 1988;52(1):30-41.

30. Shin JS, Lee Y.B. The effects of social supports on psychosocial well-being of the unemployed. Korean J Soc Welf. 1999;37:241-69.

31. Jang J. Influence that marital satisfaction and fostering stress of marriage immigrating women has on the cultural adaptation [Unpublished master's thesis]. The Graduate School of Social Development, Chung-Ang University; 2008.

32. Shaghaghi A, Bhopal RS, Sheikh A. Approaches to recruiting 'hard-to-reach' populations into research: A review of the literature. Health Promot Perspect. 2011;1(2):86-94. 
33. Cooke-Jackson A. Urban Communities as locations for health, media literacy and civic voice. In: Dezuanni M, Foth M, Mallan K, Hughes H, editors. Digital Participation through Social Living labs. Chandos Publishing; 2018. p. 207-22

34. Song YH, Kim KS, Lee SW, Choi SH. The health status including biologic exposure indices of migrant workers: Based on 2005 special health examination data. Korean J Occup Environ Med. 2008;20(2):63-73.

35. Kim, M. S. (2014). Health and medical status of migrant workers. Monthly Welfare Trends. 2014;190:15-22.

36. Wagner JA, Bermudez-Millan A, Damio G, Segura-Perez S, Chhabra J, Vergara C, et al. A randomized, controlled trial of a stress management intervention for Latinos with type 2 diabetes delivered by community health workers: Outcomes for psychological wellbeing, glycemic control, and cortisol. Diabet Res Clin Pract. 2016;120:162-170.

37. Khunti K, Camosso-Stefinovic J, Carey M, Davies M, Stone M. Educational interventions for migrant South Asians with Type 2 diabetes: a systematic review. Diabet Med. 2008;25(8):985-92.

38. United Nations. High-level political forum 2019 under the auspices of ECOSOC. 2016. https://sustainabledevelopment.un.org/hlpf/2019. Accessed 10 August 2020.

39. World Health Organization. Health 2020: a European policy framework and strategy for the 21st century. World Health Organization. Regional Office for Europe; 2013.

40. Gunasekara A, Grant S, Rajendran D. Years since migration and wellbeing among Indian and Sri Lankan skilled migrants in Australia: mediating effects of acculturation. Int J Intercult Relat. 2019;70:42-52.

\section{Figures}




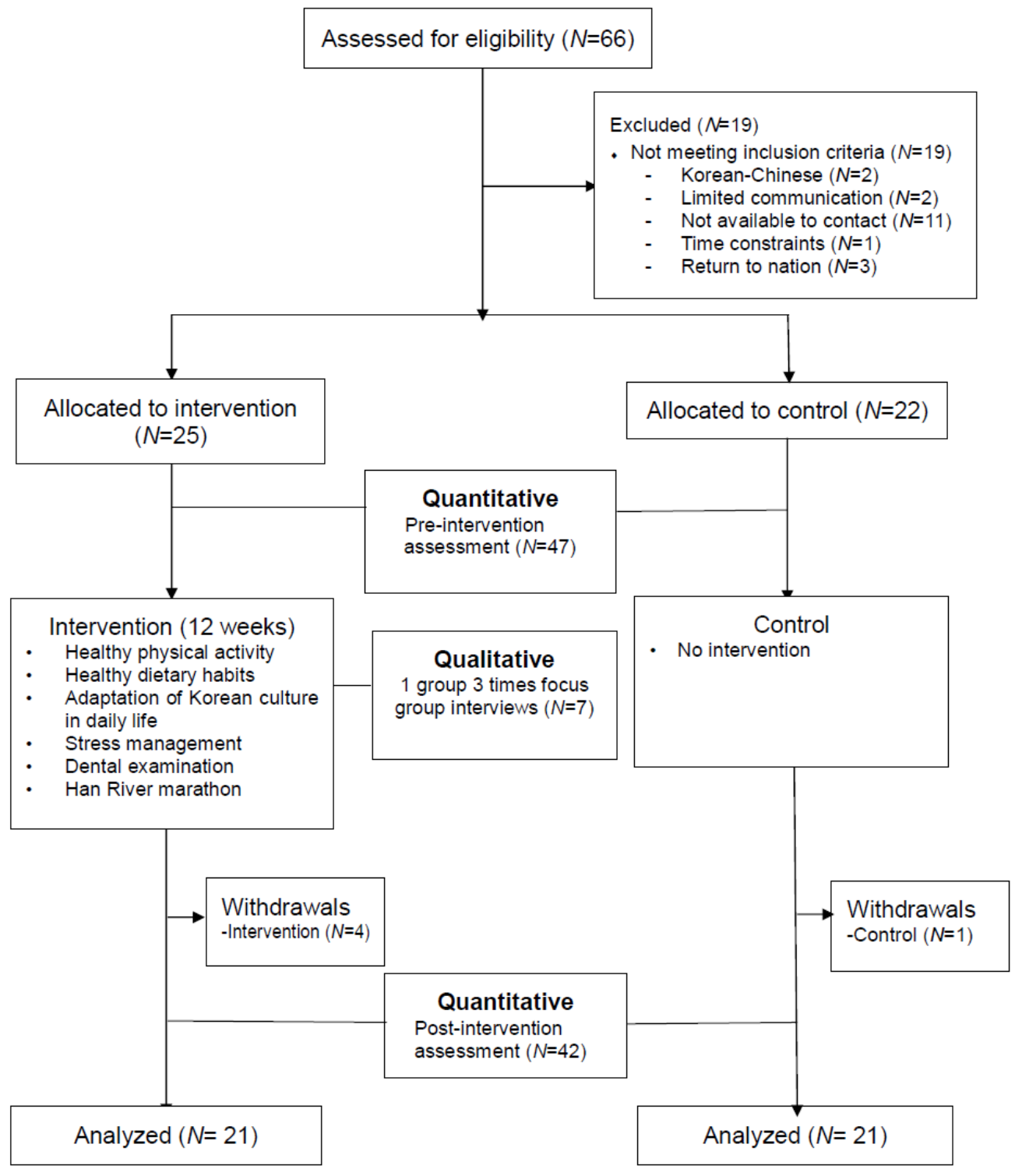

\section{Figure 1}

Intervention Design of Mixed Methods Study 


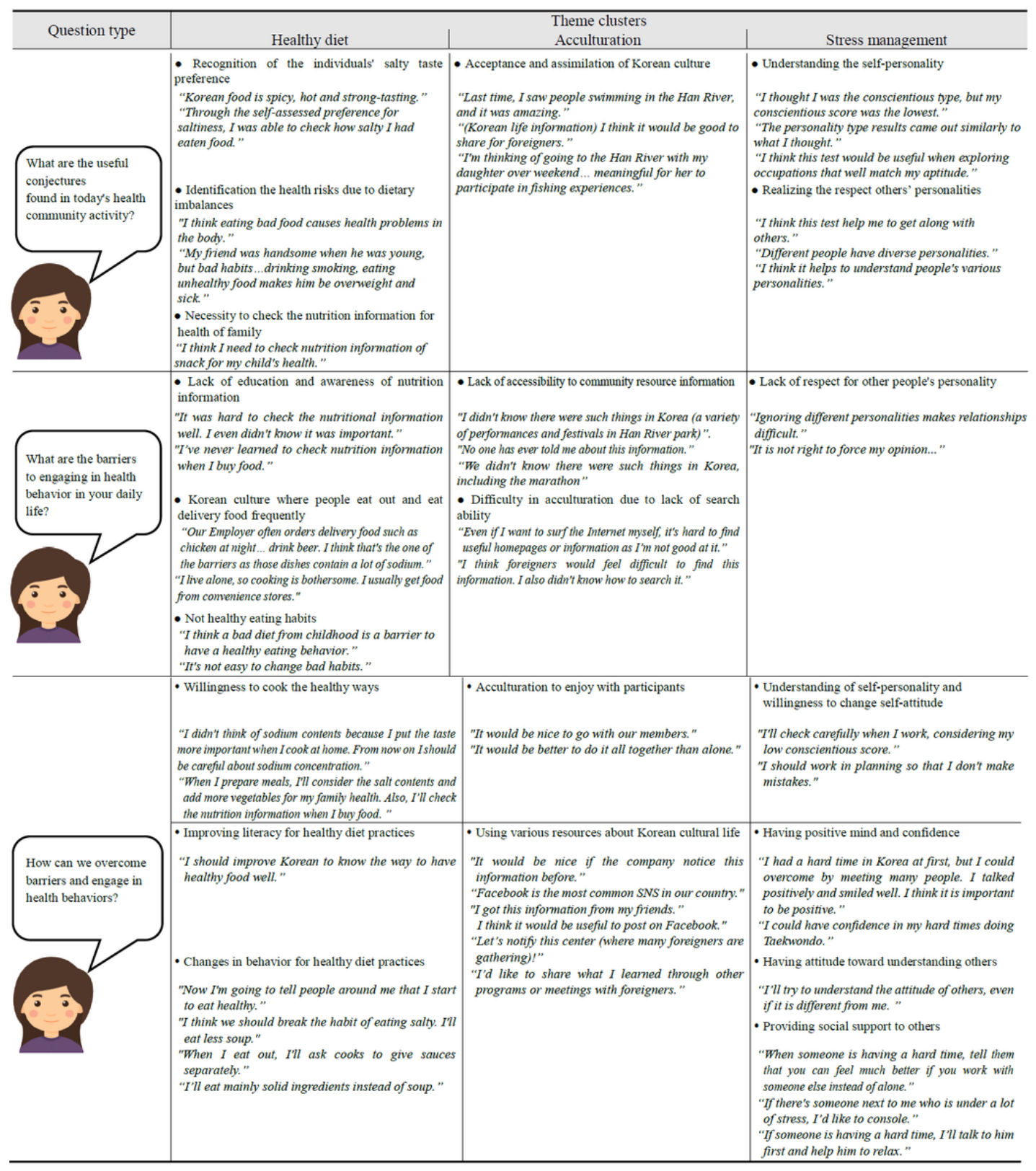

\section{Figure 2}

Analysis of Qualitative Data 